\title{
The aln Gene is Required for the Induction of the Purine Deamination Pathway in Schizosaccharomyces pombe
}

\author{
By R. FLURI ${ }^{1}$ AND J. R. KINGHORN ${ }^{1,2 *} \dagger$ \\ ${ }^{1}$ Institute of Microbiology, University of Bern, Bern CH-3013, Switzerland \\ 2 Department of Biochemistry and Microbiology, University of St Andrews, North Street, \\ St Andrews, Fife KY16 9AL, UK
}

(Received 10 April 1984; revised 27 June 1984)

Five mutants were isolated at the all 2 gene on the basis of their inability to utilize hypoxanthine as a sole source of nitrogen. These mutants failed to utilize the purines adenine, hypoxanthine, xanthine, uric acid, allantoin and allantoic acid, although they could utilize urea and ammonium. The all 2 mutants appeared to be defective in purine induction of uricase, allantoinase, allantoicase and ureidoglycollase activities but retained wild-type activity of the constitutively synthesized urease. The all 2 mutations were recessive.

\section{INTRODUCTION}

The fission yeast Schizosaccharomyces pombe is able to utilize the purines hypoxanthine and xanthine as sole nitrogen sources by degrading them to ammonium. This breakdown requires at least five enzymes (Fig. 1), namely uricase, allantoicase, allantoinase, ureidoglycollase and urease (Kinghorn \& Fluri, 1984). Unlinked mutations have been identified and characterized which result in the lack of uricase (in the urol gene), allantoinase (all 1 gene), allantoicase (ala1 gene) and urease (ure 1, ure 2 , ure 3 and ure 4 genes) activities (Fig. 1). The physiological and genetic organization of the uric acid to ammonium sequence of the pathway in $S$. pombe therefore appears to be similar to that found in filamentous fungi (Marzluf, 1981; Scazzocchio \& Gorton, 1977; Scazzocchio, 1980) and is unlike that found in the budding yeast Saccharomyces cerevisiae (Cooper, 1980). Unfortunately, the mechanisms for the conversion of hypoxanthine and xanthine to uric acid are unclear in $S$. pombe, although at least six genes are involved. Mutations in the hyp 1, hyp2, hyp 3 and hyp 4 genes result in poor growth with hypoxanthine as the sole nitrogen source but appreciable growth with uric acid and xanthine (Kinghorn \& Fluri, 1984). Poor growth of hyp 5 strains is found with both hypoxanthine and xanthine but normal growth with uric acid. The xanl mutant grows poorly on xanthine but shows wild-type growth with hypoxanthine. Although we are unable to demonstrate enzyme activity involved in the conversion of hypoxanthine or xanthine to uric acid in wild-type cells, we interpret the genetic results as follows. First, there is a 'hypoxanthine pathway' specific for hypoxanthine which is lost in hyp 1, hyp 2, hyp 3 and hyp 4 mutants. If this reaction is mediated by purine hydroxylase, several of these genes could be required for the $c n x$ cofactor. Second, there is a unique system for xanthine breakdown which may be equivalent to the Aspergillus nidulans 'alternative xanthine pathway' and which is lost in xan 1 mutants (Sealy-Lewis et al., 1978). The hyp 5 mutants on this hypothesis may encode a cofactor shared by both xanthine and hypoxanthine enzymes.

Of special interest here are the control features of the $S$. pombe purine breakdown systems. Uricase, allantoinase, allantoicase and ureidoglycollase are highly inducible and this induction

† Correspondence should be sent to the St Andrews address.

Abbreriation: MIN, minimal medium. 


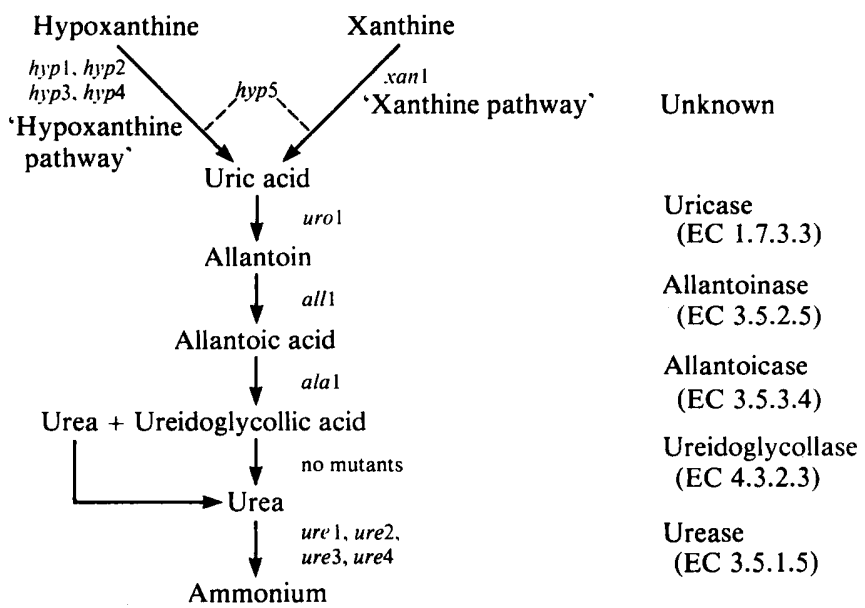

Fig. 1. Route of purine catabolism in Schizosaccharomyces pombe.

is brought about by purines (Fluri \& Kinghorn, 1985). It is not yet known, except for uricase, whether control is at the level of transcription or translation or both. In vitro translation studies show that, in the case of uricase, induction control is at the level of transcription (R. Fluri, unpublished work). In contrast, the regulation of urease, the final step in the pathway, is constitutive (Fluri \& Kinghorn, 1985). Examination of the various classes of mutants that do not utilize purines has permitted the identification of the true inducers of the pathway. These studies suggest that control is brought about by sequential induction of the enzymes by various intermediates of the pathway (Fluri \& Kinghorn, 1985). Uricase is induced by uric acid, allantoinase and allantoicase by allantoic acid and ureidoglycollase by ureidoglycollic acid. These conclusions raise the following question. If there are three distinct inducers required for the induction of four unlinked structural genes, how many regulatory genes are required to mediate control by these co-inducer molecules? The present study suggests that a single gene (all2) is involved.

\section{METHODS}

Media and growth conditions. The sporulation medium used for genetical crosses was malt extract agar; yeast extract/glucose was used as complete medium for routine growth, as described by Gutz et al. (1974); minimal medium (MIN) was $1 \%(w / v)$ glucose, Difco yeast nitrogen base (without amino acids and ammonium) supplemented with a nitrogen source.

Genetical analysis. The procedures described by Leupold (1970) and Gutz et al. (1974) were followed. All mutants were generated in standard wild-type strains 972 (mating type $\mathrm{h}^{-}$) or 975 (mating type $\mathrm{h}^{+}$). Diploids were constructed in the way described by Gygax \& Thuriaux (1984). First, a double mutant was constructed between all2-1 and $c d c 12-12$ in an $\mathrm{h}^{-}$mating type background. A second double mutant was constructed between $c d c 1-7$ and the purine mutation under study, in a mat 2 mating type background. Diploids were selected on the basis of complementation of $c d c$ mutations (normal growth at $35^{\circ} \mathrm{C}$ ) and screened for their ability to grow with hypoxanthine as sole nitrogen source.

Grow'th tests. Responses were measured on MIN containing test substance ( $2 \mathrm{mM})$ as sole nitrogen source. Tests were done on $10 \mathrm{ml}$ and $50 \mathrm{ml}$ liquid cultures in conical flasks which were reciprocally shaken at $30^{\circ} \mathrm{C}$ for periods up to $48 \mathrm{~h}$. Growth was analysed photometrically in diluted suspensions. One optical density unit $\left(\mathrm{OD}_{530}\right)$ was equivalent to $2 \cdot 2 \times 10^{7}$ cells $\mathrm{ml}^{-1}$. The incubation was started with a reading of $\mathrm{OD}_{530} 0 \cdot 1$.

Growth conditions for enzyme assays. The wild-type strain (972) and mutant strains were grown for approximately $15 \mathrm{~h}$ in MIN plus $1 \mathrm{~mm}$-ammonium tartrate ( $2 \mathrm{~mm}$-ammonia) as sole nitrogen source. After $15 \mathrm{~h}\left(\mathrm{OD}_{530} 2-3\right)$, by which time the ammonium nitrogen source was depleted (R. Fluri, unpublished work), a purine inducer ( $2 \mathrm{mM})$, plus $2 \mathrm{~mm}$ glutamate as a neutral nitrogen source, was added and the cells were re-incubated for $5 \mathrm{~h}$ (Fluri \& Kinghorn, 1985). 
Permeabilization. Cell suspensions were centrifuged, washed twice in Tris buffer $(0.1 \mathrm{M}, \mathrm{pH} \mathrm{8.0)}$ and resuspended in $1 \mathrm{ml}$ of the same buffer plus $0.05 \%(\mathrm{v} / \mathrm{v})$ Triton $\mathrm{X}-100$. The cell density was adjusted to $\mathrm{OD}_{530} 14 \cdot 7$ $\left(10 \mathrm{mg}\right.$ wet $\left.\mathrm{wt} \mathrm{ml}^{-1}\right)$. This sample was frozen quickly at $-70^{\circ} \mathrm{C}$ in a dry ice/acetone bath. After $15 \mathrm{~min}$ the cell suspension was thawed rapidly in a water bath at $35^{\circ} \mathrm{C}$ and permeabilized cells were stored at $0{ }^{\circ} \mathrm{C}$. For each assay $0 \cdot 1 \mathrm{mg}$ wet wt permeabilized cells $\left(\mathrm{OD}_{530} 0 \cdot 147\right)$ were used.

Enzymology. Uricase activity was assayed by measuring the rate of disappearance of uric acid from the decline in absorbance at $292 \mathrm{~nm}$ in the presence of permeabilized cells in $2 \mathrm{ml}$ glycine/ $\mathrm{NaOH}$ buffer $(0 \cdot 1 \mathrm{M}, \mathrm{pH} 8 \cdot 8)$. The activities of allantoinase, allantoicase and ureidoglycollase were determined by the method of Vogels \& van der Drift (1970). Urease activity was assayed by measuring the production of ammonium ions. The ammonium ion concentration was measured directly by the colorimetric procedure of Muftic (1964) or indirectly using a commercial assay system involving glutamate dehydrogenase coupled with NADH oxidation.

Isolation of mutant strains that did not utilize purines. Haploid cells of $S$. pombe from four yeast extract/glucose agar 4-d-old slants were suspended in $4 \mathrm{ml}$ sodium acetate buffer $(0.2 \mathrm{M}, \mathrm{pH} 5 \cdot 0)$, pelleted by centrifugation and resuspended in $1.5 \mathrm{ml}$ acetate buffer. The cells were counted using a Thoma counting chamber. A portion of this suspension, containing $3 \times 10^{8}$ cells $\mathrm{ml}^{-1}$, was used for the mutagenesis treatment. $N$-methyl- $N^{\prime}$-nitro- $N$ nitrosoguanidine (NTG) was added at a concentration of $2 \mathrm{mg} \mathrm{ml}^{-1}$ as described by Adelberg et al. (1965) and standardized for $S$. pombe according to Leupold (1970). A 13 min treatment at $30{ }^{\circ} \mathrm{C}$ resulted in a $60 \%$ kill. Since nystatin selection is used routinely for the isolation of mutants from Saccharomyces cerevisiae (Snow, 1966) we used a similar approach for $S$. pombe. The mutagenized culture was allowed to recover by overnight incubation in yeast extract/glucose broth, washed twice with physiological saline and resuspended in $200 \mathrm{ml}$ MIN medium with hypoxanthine as sole nitrogen source. After a $5 \mathrm{~h}$ adaptation period, the suspension (containing $2.5 \times 10^{5}$ viable cells $\left.\mathrm{ml}^{-1}\right)$ was treated with nystatin $\left(10 \mu \mathrm{g} \mathrm{ml}^{-1}\right)$ and re-incubated for $4 \mathrm{~h}$. The culture was washed twice in physiological saline and resuspended in $2 \mathrm{ml}$ saline. This nystatin treatment reduced the $S$. pombe population by $99.5 \%$. Appropriate dilutions were spread on to MIN-ammonium plates to give approximately 200 colonies per plate. Putative hypoxanthine non-utilizing mutants were tested for growth on MIN-hypoxanthine plates. The strains which grew on $\mathrm{MIN}$-ammonium but failed to grow on MIN-hypoxanthine were kept for biochemical and genetical analysis. This procedure yielded mutants in a number of genes involved in purine breakdown (Kinghorn \& Fluri, 1984) at a frequency of approximately $1 \%$, representing an enrichment of 30-40-fold. Two all 2 mutants were initially isolated and three from a later experiment.

\section{RESULTS}

\section{Isolation and genetical characterization of all 2 strains}

Five mutants were isolated on the basis of their inability to grow on hypoxanthine as sole nitrogen source. They grew as wild-type with ammonium and with a range of other nitrogen sources, showing that the mutant defects were limited to purine breakdown. All purine defective strains were re-isolated and backcrossed to the wild-type for genetical purification and introduction of the opposite mating type. all 2 mutants are unable to utilize hypoxanthine, uric acid or allantoin as sole nitrogen source on solid medium but can utilize allantoic acid, urea and ammonium (see below for more definitive data from liquid culture). Analysis of the segregation of these characteristics in tetrads showed that all 2 mutations are monogenic.

Recombination analyses by the criss-cross method suggested that all five mutants were very closely linked. In order to confirm the allelism found by the criss-cross technique, complementation tests were set up between all2-1 and other all 2 alleles. No complementation occurred between all2-1 and all other all 2 alleles. In contrast, all 2 and all 1 mutants, although having a similar phenotype on solid media, complemented one another and are unlinked as judged by the criss-cross test.

The all2-1 mutant was crossed to representative alleles at other genes involved in purine catabolism. No significant linkage was detected in crosses with urol, ala1, ure1, ure 2, ure 3, ure4, hyp 2, hyp3, hyp 4 or hyp 5 strains. In striking contrast, the criss-cross test showed that all 2 mutants were closely linked with hyp 1-50. Free spore analysis was carried out between hyp 1-50 and all2-1 with 2000 progeny examined. Two prototrophs were recovered, showing a recombination frequency of $0.2 \%$. However, this could be an over-estimation since useful flanking markers were not available. To test for allelism, hyp 1-50 was crossed to three alleles at the all 2 locus. Diploids were selected on the basis of complementation of the $c d c$ mutation (normal growth at $35^{\circ} \mathrm{C}$ ) and screened for their ability to grow with hypoxanthine as sole nitrogen source. To 

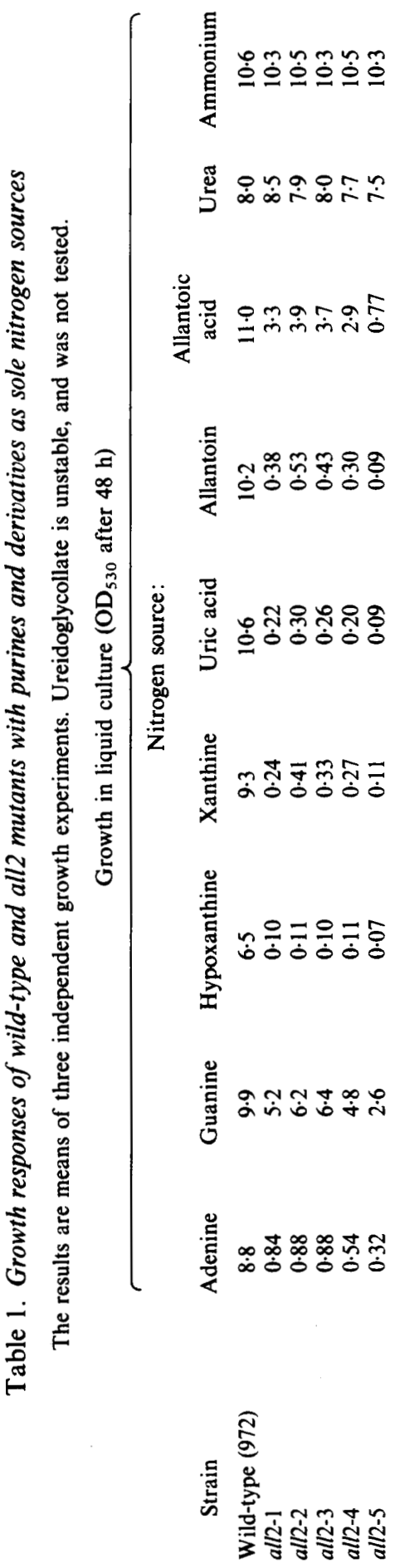

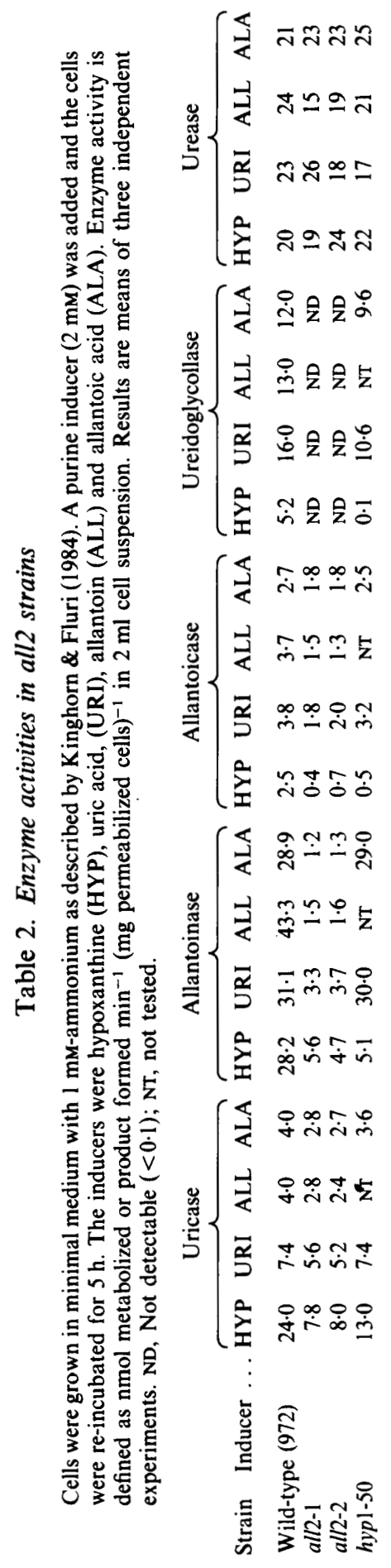


ensure that the strains under test were in fact diploids they were haploidized using $\mathrm{m}$ fluorophenylalanine (Kohli et al., 1977) and the various genotypes recovered. None of the constructed diploids was able to grow on hypoxanthine showing that hyp 1-50 and all 2 mutations are allelic.

\section{Growth responses of all 2 strains}

The ability of all 2 mutants to utilize purines and their intermediates in liquid culture is shown in Table 1. The mutants grew poorly compared with wild-type in the presence of adenine, hypoxanthine, xanthine, uric acid and allantoin. They grew better, although not as well as wildtype, with guanine and allantoic acid and grew as wild-type with urea and unrelated nitrogen sources, such as alanine, glutamate, glutamine, uracil, arginine and ammonium. It is significant that hyp1-50 grows as wild-type with xanthine, uric acid, allantoin, allantoic acid, urea and ammonium as sole nitrogen sources (Kinghorn \& Fluri, 1984). Its only defect appears to be its inability to utilize hypoxanthine.

\section{Enzyme activities in all 2 and hyp1-50 strains}

Table 2 shows the induction of uricase, allantoinase and ureidoglycollase activities in two representative all 2 strains compared with wild-type induction. It is clear that induction of uricase was affected by all 2 mutations. This was particularly marked with hypoxanthine, which has to be converted to uric acid, the true inducer, before induction can occur (Fluri $\&$ Kinghorn, 1985). all2 mutants show less reduction of activity with uric acid itself as the inducer. Allantoinase levels were reduced in all 2 strains treated with hypoxanthine, allantoin, allantoic acid and uric acid. Similarly, allantoicase and ureidoglycollase levels were low under the conditions tested. Therefore, all four inducible purine catabolic activities are reduced in all 2 strains grown under the four conditions. On the other hand, urease activity, which is constitutive in the wild-type, was completely unaffected by all 2 mutations. Heterozygous diploids of wildtype and the various all 2 alleles showed wild-type activity as well as wild-type growth with purines, indicating that all 2 mutations are completely recessive.

In contrast, hyp1-50 has markedly reduced inducible levels of uricase, allantoinase, allantoicase and ureidoglycollase when grown on hypoxanthine only and, unlike all 2 mutations, retains almost wild-type levels of all four enzymes with the other inducers. Urease activity is wild-type in hyp1-50 even when grown on hypoxanthine.

\section{DISCUSSION}

We have identified a gene which may be involved in regulation of the purine catabolic pathway. Mutations in the all 2 gene pleiotropically affect inducible enzymes of the pathway, namely uricase, allantoinase, allantoicase and ureidoglycollase. The systems required for xanthine and hypoxanthine could also be affected by all 2 mutations. However, this cannot be tested directly as we were unable to assay purine hydroxylase activity although several assay methods were used (Kinghorn \& Fluri, 1984). In contrast, the constitutive enzyme urease is insensitive to all 2 mutations. The all 2 gene affects, therefore, the activity of four separate enzymes which are sequentially induced by three different co-inducer molecules, i.e. uricase by uric acid, allantoinase and allantoicase by allantoic acid, and ureidoglycollase by ureidoglycollic acid. The fact that none of the enzymes is induced by the respective inducer suggests a common mechanism. If all 2 is a control gene specifying a regulatory element, its product must interact with these various inducer molecules. Significantly, this would imply that the control element possesses a site (or sites) which recognizes all three, and possibly other, inducer molecules. If the all2 product has a regulatory function, this might be at the level of transcription such as has been shown for uricase by in vitro mRNA assays (R. Fluri, unpublished work). However, it must be stressed that this conclusion represents the simplest interpretation of the data and there are alternative explanations for the role of all 2 in purine catabolism. For example, all 2 may code for a common component of all four regulated enzyme systems, although this seems less likely. Another possibility is that catalytic components required for hypoxanthine and xanthine play a 
direct role in the regulation of the rest of the pathway. all2, on this hypothesis, may encode a structural component for a purine hydroxylase. Inducer exclusion can be ruled out since all 2 mutants have appreciable wild-type transport rates (R. Fluri, unpublished work).

Finally, the basis of hypl-50 (which will be referred to in future as all2-50) is puzzling but must obviously be a very specific change which appears to affect the unidentified catalytic step which is responsible for hypoxanthine conversion to uric acid. Low activity of the four inducible enzymes, namely uricase, allantoinase, allantoicase and ureidoglycollase, is found in hyp 1-50 when treated with hypoxanthine only. This secondary effect is likely to be due to the primary defect - the inability of the strain to convert hypoxanthine into the various true inducers. We plan to isolate the all 2 locus by molecular cloning techniques to resolve these uncertainties.

The all 2 gene may be equivalent in certain respects to the Aspergillus nidulans uaY gene which mediates uric acid induction of the enzymes required for purine breakdown (Scazzocchio \& Gorton, 1977). There is considerable evidence that $u a \mathrm{Y}$ is a regulatory gene with a positive mode of control, although no $u a \mathrm{Y}^{\mathrm{c}}$ mutations have been found. Similarly, despite strenuous efforts, constitutive mutants at the all 2 gene have not been isolated.

J. R. K. is indebted to the Royal Society for a Research Fellowship under the European Exchange Programme. We also acknowledge funds provided by the Swiss National Science Foundation to R. F. We thank Frau Edenharder for excellent technical assistance.

\section{REFERENCES}

Adelberg, E. A., Mandel, M. \& Chen, G. C. C. (1965). Optimal conditions for mutagenesis by $N$ methyl- $N^{\prime}$-nitro- $N$-nitrosoguanidine in Escherichia coli K12. Biochemical and Biophysical Research Communications 18, 788-793.

COOPER, R. G. (1980). Selective gene expression and intracellular compartmentation: two means of regulating nitrogen metabolism in yeast. Trends in Biochemical Sciences December, 1-4.

FLURI, R. \& KINGHORN, J. R. (1985). The induction of purine catabolism in Schizosaccharomyces pombe. Current Genetics (in the Press).

Gutz, H., Heslot, H., Leupold, U. \& Loprieno, N. (1974). 'Schizosaccharomyces pombe'. In Handbook of Genetics, vol. 1, pp. 395-446. Edited by R. C. King. New York: Plenum Press.

Gygax, A. \& Thuriaux, P. (1984). A revised chromosome map of the fission yeast Schizosaccharomyces pombe. Current Genetics 8, 85-92.

Kinghorn, J. R. \& Fluri, R. (1984). Genetic studies of purine breakdown in the fission yeast Schizosaccharomyces pombe. Current Genetics 8, 99-105.

Kohli, J., Hottinger, H., Munz, P., Strauss, A. \& Thuriaux, P. (1977). Genetic mapping in Schizosaccharomyces pombe by mitotic and meiotic and induced haploidisation. Genetics 87, 471-489.

LEUPOLD, U. (1970). Genetical methods for Schizo- saccharomyces pombe. Methods in Cell Physiology 4, 169-177.

Marzluf, G. A. (1981). Regulation of nitrogen metabolism and gene expression in fungi. Microbiological Review's 45, 437-461.

MufTIC, M. K. (1964). A new phenol-hypochlorate reaction for ammonia. Nature, London 201, 622-623.

Scazzocchio, C. (1980). The genetics of molybdenumcontaining enzymes. In Molybdenum and Molybdenum Containing Enzlmes, pp. 487-516. Edited by M. P. Coughlan. New York: Pergamon Press.

Scazzocchio, C. \& GorTon, D. (1977) Regulation of purine breakdown. In Genetics and Physiology of Aspergillus, pp. 255-265. Edited by J. E. Smith \& J. A. Pateman. London: Academic Press.

Sealy-Lewis, H. M., Scazzocchio, C. \& Lee, S. (1978). A mutation defective in the xanthine alternative pathway of Aspergillus nidulans. Its use to investigate the specificity of $u a \mathrm{Y}$ mediated induction. Molecular and General Genetics 164, 303308.

SNow, R. (1966). An enrichment method for auxotrophic yeast mutants using the antibiotic 'nystatin'. Nature, London 211, 206-207.

Vogels, G. D. \& VAN DER DRIFT, C. (1979). Differential analysis of glyoxylate derivatives. Analytical Biochemistry 33, 143-157. 